\title{
What Causes Pulmonary Arterial Hypertension in Down Syndrome With Congenital Heart Disease?
}

\author{
Susumu Hosokawa, MD, PhD; Rebecca R. Vanderpool, PhD; Taku Ishii, MD; \\ Mitsunori Nishiyama, MD, PhD; Shozaburo Doi, MD, PhD
}

D own syndrome (DS) is the most common chromosomal abnormality. A recent study showed that its incidence is 1 in $319-1,000$ live births. ${ }^{1}$ Approximately half of all newborns with DS have congenital heart disease (CHD): ${ }^{2}$ atrioventricular septal defect (AVSD: $45 \%$ ), ventricular septal defect (VSD: $35 \%$ ), secundum atrial septal defect ( $8 \%$ ), and tetralogy of Fallot (4\%). ${ }^{3}$

DS strongly associates with pulmonary arterial hypertension (PAH). Patients with DS and CHD are thought to develop PAH more rapidly than non-DS patients with CHD. ${ }^{4}$ In patients with DS and severe pulmonary hypertension, cardiologists need to determine whether there are surgical indications when they perform the cardiac catheterizations. In those cases, the Heath-Edwards (H-E) classification, and in Japan the index of pulmonary vascular disease (IPVD) ${ }^{5}$ proposed by Yamaki and Tezuka, ${ }^{6}$ determined from a lung biopsy play an important role in assessing the need for surgery. The IPVD is an important measure to quantify the severity of pulmonary arteriopathy lesions. Yamaki showed that surgery was indicated in patients with a VSD and DS when the IPVD was $\leq 2.1$, whereas VSD patients without DS had an IPVD $\leq 2.0 .{ }^{7}$ Pulmonary wedge angiography in patients with DS shows there are fewer branches and capillaries, and increased tortuosity of the vessels than in patients without the chromosomal abnormality. ${ }^{8}$ These changes in the pulmonary vascular structure suggest that patients with DS may have more progressive PAH than patients without DS.

In this issue of the Journal, Masaki et $\mathrm{al}^{9}$ evaluate the severity of the histopathological changes in pulmonary arteriopathic lesions in patients with simple VSD or AVSD with and without DS. The severity of intimal lesions was assessed using the H-E classification and IPVD score. In their overall cohort, the systolic pulmonary arterial pressure to systolic systemic blood pressure ratio (sysPp/Ps) was higher in patients with DS than in those without DS, but there was no significant difference in pulmonary vascular resistance $(\mathrm{Rp})$ between groups. In addition, every hemodynamic parameter in the propensity score-matched cohort did not show any significant difference between groups.

Notably, the severity of the pulmonary arteriopathic lesions did not differ between the groups in terms of the

\section{Article p 1682}

H-E classification and IPVD score. Furthermore, earlier progression of histopathological changes in pulmonary arteriopathy could not be proved in patients with DS despite accounting for age in the histological findings. Previous papers have reported no difference in the incidence of PAH between patients with DS and those without DS. Vázquez-Antona et al showed that results of a hemodynamic study of pulmonary hypertension and vasodilation test with oxygen were similar for patients with DS and those without the chromosomal abnormality, ${ }^{10}$ though they did not examine histological findings.

Cohort selection, other types of respiratory problems and anatomic variability are other reasons that could explain the results in the current study by Masaki et al. Patients who had undergone pulmonary artery banding were excluded from this study, which might cause underestimation of the severity of pulmonary arteriopathy in the group of patients with DS. Other factors could be contributing to the pathogenesis of PAH in patients with DS. Pandit et al suggest there are many respiratory problems in both the upper and lower airways of children with DS.11 Anatomic characteristics in DS such as large tongue, small hypopharynx, pharyngeal hypotonia, tonsils and adenoid enlargement, laryngomalacia, and tracheomalacia cause upper airway problems. In addition, gastroesophageal reflux, immunologic dysfunction, tracheal bronchus, airway malacia, CHD, and pulmonary hypoplasia cause recurrent lower airway diseases. ${ }^{11}$ Cooney and Thurlbeck showed that 6 of 7 patients with DS had hypoplastic lungs, and 5 had CHD. ${ }^{12}$ Patients with DS have a decreased number of alveoli in relation to acini, enlarged alveoli and alveolar ducts, and a smaller alveolar surface area, which can lead to exacerbation of pulmonary hypertension in DS. ${ }^{12}$ Bush et al investigated the histologic features of impaired development of the lung vasculature and alveoli and the presence of intrapulmonary bronchopulmonary anastomoses (IBA) in patients with DS using specimens obtained from autopsy. ${ }^{13}$ These patients had frequent histologic evidence of impaired lung alveolar and vascular development,

The opinions expressed in this article are not necessarily those of the editors or of the Japanese Circulation Society.

Received April 22, 2018; accepted April 25, 2018; released online May 12, 2018

Department of Pediatrics, Tokyo Medical and Dental University, Tokyo (S.H., T.I., M.N., S.D.), Japan; Division of Translational and Regenerative Medicine, The University of Arizona College of Medicine, Tucson, AZ (S.H., R.R.V.), USA

Mailing address: Susumu Hosokawa, MD, PhD, Department of Pediatrics, Tokyo Medical and Dental University, 1-5-45 Yushima, Bunkyo-ku, Tokyo 113-8510, Japan. E-mail: shosokawa.ped@tmd.ac.jp

ISSN-1346-9843 All rights are reserved to the Japanese Circulation Society. For permissions, please e-mail: cj@j-circ.or.jp 
including the presence of IBA and pulmonary hypertension, which may worsen gas exchange in DS. ${ }^{13}$

Questions remain regarding PAH in patients with DS. Many previous studies have shown that patients with CHD in DS tend to develop PAH faster than patients without DS. On the other hand, some studies, including that by Masaki et al, ${ }^{9}$ indicate no significant difference in the progression of PAH between patients with and without DS. Because of the additional factors that contribute to the pathogenesis of pulmonary hypertension in patients with DS it is difficult to draw definitive conclusions.

More information is needed to determine in which cases there are surgical indications and what causes PAH in patients with DS. The current histological evaluation methods such as the H-E classification and IPVD score are a good start but additional biomarkers are needed. Galambos et al focused on some anti-angiogenic genes that are encoded by human chromosome 21.14 They found that COL18A1 (endostatin), COL4A3 (tumstatin), TIMP3 (tissue inhibitor of metalopeptidase 3), and APP (amyloid protein precursor), all of which are lung anti-angiogenic factors, were upregulated in subjects with DS. ${ }^{14}$ These genes impaired lung vascular growth and signaling and might contribute to the high risk for PAH during infancy. ${ }^{14}$ Huang et al proposed some pathological biomarkers. ${ }^{15}$ In irreversible PAH, they found increased expression of caveolin-1, filaminA, and cathepsin D and decreased glutathione S-transferase mu 1 (GSTM1) from a lung biopsy, which could be used as an expression profile to predict irreversible PAH caused by CHD. ${ }^{15}$ Hopefully, new and improved methods to evaluate the histological findings, and the discovery of new biomarkers will lead to better understanding of the pathogenesis of PAH in patients with DS.

\section{Conflict of Interest}

No potential conflicts of interest are disclosed.

\section{References}

1. Wiseman FK, Alford KA, Tybulewic VLJ, Fisher EM. Down syndrome: Recent progress and future prospects. Hum Mol Genet 2009; 18: R75-R83.

2. Patterson D. Molecular genetic analysis of Down syndrome. Hum Genet 2009; 126: 195-214.

3. Roizen NJ, Patterson D. Down's syndrome. Lancet 2003; 361: $1281-1289$.

4. Hawkins A, Langton-Hewer S, Henderson J, Tulloh RM. Management of pulmonary hypertension in Down syndrome. Eur J Pediatr 2011; 170: 915-921.

5. Heath D, Edwards JE. The pathology of hypertensive pulmonary vascular disease: A description of six grades of structural changes in the pulmonary arteries with special reference to congenital cardiac septal defects. Circulation 1958; 18: 533-547.

6. Yamaki S, Tezuka F. Quantitative analysis of pulmonary vascular disease in complete transposition of the great arteries. Circulation 1976; 54: 805-808.

7. Yamaki S. Pulmonary vascular disease associated with pulmonary hypertension in 445 patients: Diagnosis from lung biopsy and autopsy. Gen Thorac Cardiovasc Surg 2013; 61: 24-31.

8. Fujiyama J, Yoshida Y, Ogata H, Ohara T, Murata Y, Abe J, et al. Assessment of pulmonary vascular bed by pulmonary wedge angiography in patients with Down syndrome. Shinzo 1988; 20: 823-829 (in Japanese).

9. Masaki N, Saiki Y, Endo M, Maeda K, Adachi O, Akiyama M, et al. Is trisomy 21 a risk factor for rapid progression of pulmonary arteriopathy?: Revisiting histopathological characteristics using 282 lung biopsy specimens. Circ J 2018; 82: 1682-1687.

10. Vázquez-Antona CA, Lomeli C, Buendia A, Vargas-Barron J. Pulmonary hypertension in children with Down's syndrome and congenital heart disease: Is it really more severe? Arch Cardiol Mex 2006; 76: 16-27 (in Spanish).

11. Pandit C, Fitzgerald DA. Respiratory problems in children with Down syndrome. J Paediatr Child Health 2012; 48: E147-E152.

12. Cooney TP, Thurlbeck WM. Pulmonary hypoplasia in Down syndrome. N Engl J Med 1982; 307: 1170-1173.

13. Bush D, Abman SH, Calambos C. Prominent intrapulmonary bronchopulmonary anastomoses and abnormal lung development in infants and children with Down syndrome. J Pediatr 2017; 180: $156-162$

14. Galambos C, Minic AD, Bush D, Nguyen D, Dodson B, Seedorf $\mathrm{G}$, et al. Increased lung expression of anti-angiogenic factors in Down syndrome: Potential role in abnormal lung vascular growth and the risk for pulmonary hypertension. PLoS One 2016; 11: e0159005.

15. Huang L, Li L, Hu E, Chen G, Meng X, Xiong C, et al. Potential biomarkers and targets in reversibility of pulmonary arterial hypertension secondary to congenital heart disease: An explorative study. Pulm Circ 2018; 8: 1-12. 